\title{
Treatment of experimental pulmonary embolism by main pulmonary arterial infusion of a fibrinolytic agent ${ }^{1}$
}

\author{
DA N I O I. MAGTIRA, M. WELLS, R. LLA MAS, \\ R. CLARK, A N D G. L. B A U M
}

From the Cardiopulmonary Laboratory, Veterans Administration Hospital, Coral Gables, Florida, and the Department of Medicine, University of Miami School of Medicine, Miami, Florida

\begin{abstract}
The continuous instillation of plasmin (Thrombolysin) directly into the main pulmonary artery of greyhounds, using one half of the systemic doses, was found to be more effective in accelerating or enhancing in vivo lysis of artificially produced pulmonary emboli than systemic intravenous infusion. Animals which received direct pulmonary arterial instillations of Thrombolysin showed remarkably few macroemboli. By light microscopy, these clots showed zones of lysis mainly in the central portions of the clots. We suggest that the flow-guided catheterization of the main pulmonary artery used in this study on greyhounds can be applied to critically ill human subjects, as shown by earlier studies (Magtira, Winkler, Duprey, and Baum, 1966). Continuous infusions of thrombolytic agents directly into the main pulmonary artery might then offer an effective therapy for human pulmonary embolism, especially during that critical period following a massive or submassive embolic episode.
\end{abstract}

Despite heparin anticoagulation and the continued refinements of surgical intervention, acute thromboembolism remains a frequent and distressing cause of morbidity and mortality in populations of the Western world, particularly in the middle-aged and the elderly. Pulmonary embolism is common and remains a challenge: it is one of the common causes of death in hospital, has been estimated to cause 47,000 deaths per year in the United States of America, and is the third most common cause of death at the Peter Bent Brigham Hospital (Dalen and Dexter, 1967).

Pulmonary embolism may give widely varying effects in different patients. It can be silent without any detectable sign or symptom. A patient can have only transient signs and symptoms without pulmonary infarction or cor pulmonale. Pulmonary infarction can occur with or without acute cor pulmonale, and acute cor pulmonale can occur with or without pulmonary infarction. Lastly, a patient can collapse and die within a short period of time, too soon for anticoagulants to be effective. The patient may be desperately ill and surgical intervention not feasible.

'Portions of this paper were presented at the National Meeting (Pulmonary Section) of the American Federation for Clinical Research, Atlantic City, New Jersey, 30 April 1967
Until more is known about the exact pathogenesis of pulmonary embolism, thrombolytic agents may prove the best way to treat this disorder. Numerous substances have been employed in the past with the purpose of enhancing or supplementing in vivo fibrinolysis, but so far there has been inadequate information about these thrombolytic agents.

Here we have studied direct continuous instillation of a fibrinolytic agent into the main pulmonary artery in enhancing or accelerating clot lysis in vivo in greyhounds with artificially induced pulmonary embolism.

\section{MATERIALS AND METHODS}

Thrombolysin $^{2}$ was used in this study. This compound is a streptokinase-activated human plasminogen preparation. It exhibits both profibrinolysinactivator and fibrinolytic properties, although its lytic properties have been attributed mainly to its streptokinase content. Each Merck, Sharp, and Dohme ? (M.S.D.) unit of Thrombolysin is the amount that $T$ will lyse a standard fibrin clot in 10 minutes under standard conditions of $p \mathrm{H}$, temperature, and concentration. The M.S.D. unit represents a house standard

2Supplied as Lyovac Thrombolysin (fibrinolysin-human) by Merck, Sharp, and Dohme Laboratories (West Point, Pennsylvania) 
which has been used throughout the trial and early marketing periods in conjunction with a standard manufacturing procedure to assure consistency of the produce. There is at present nu National Institute of Health approved fibrinolytic unit, so that it is not possible to relate this M.S.D. house standard with other fibrinolytic units using different assigned unitage. Further details regarding Thrombolysin are available on request from the manufacturer.

Twenty-four male greyhounds of comparable weights $(65 \mathrm{lb}$., $29 \mathrm{~kg}$.) were divided into three groups of eight each. Each animal was anaesthetized using Dial with Urethane Solution. ${ }^{3}$

Each animal was embolized with $130 \mathrm{ml}$. of preformed, autologous, venous clots ( 24 hours old) given in 10-20 ml. amounts over a period of 30 minutes. Preliminary trials in 60 male greyhounds (average weight $65 \mathrm{lb}$., $29 \mathrm{~kg}$.) revealed that if this total amount of $130 \mathrm{ml}$. was exceeded, the animals would die. Venous blood was collected, using aseptic techniques. from a femoral vein into sterile rubber tube coils (internal diameter $4 \mathrm{~mm}$.) approximately 26 hours before each study. The coils, appropriately marked to $250 \mathrm{ml}$., were filled with venous blood and left standing at room temperature. Generous amounts of clots morphologically resembling red thrombi were obtained in this manner. Each animal was embolized by connecting the coils to a glass cannula (internal diameter $4 \mathrm{~mm}$.) inserted securely into a jugular vein. Clots were introduced by normal saline solution displacement (carefully measured) from the distal end of the coils.

Fragmentation of the introduced macroemboli probably occurred prior to entry into smaller pulmonary arteries and probably occurred in the right heart as suggested by previous studies (Wessler, Freiman, Ballon, Katz, Wolff, and Wolf, 1961).

After introduction of the total amount $(130 \mathrm{ml}$.), sustained tachycardia, tachypnoea, and pulmonary artery hypertension occurred.

The dogs were divided into groups as follows:

GROUP 1 These animals were given dextrose $5 \%$ in water solution continuously for four hours directly into the main pulmonary artery via a flow-guided catheter.

GROUP 2 Thrombolysin in dextrose $5 \%$ in water solution was given to these animals continuously for four hours. Peripheral hind leg veins were used. The doses were 100,000 M.S.D. units for the first hour and 50,000 M.S.D. units per hour for the succeeding three hours.

GROUP 3 This group received Thrombolysin in dextrose $5 \%$ in water solution continuously for four hours directly into the main pulmonary artery via a flow-guided catheter. The doses were 50,000 M.S.D.

${ }^{3}$ Ciba Corporation, Summit, New Jersey. Each millilitre contains $100 \mathrm{mg}$. allobarbital, $400 \mathrm{mg}$. urethane, $400 \mathrm{mg}$. monoethylurea, and purified water q.s. Dose: $0.6 \mathrm{ml} . / \mathrm{kg}$. body weight units for the first hour and 25,000 M.S.D. units per hour for the succeeding three hours.

Opacification of the pulmonary arteries was obtained by selective pulmonary arteriography using $35 \mathrm{ml}$. of AngioConray ${ }^{4}$ delivered through the flowguided catheter by power injection (pressure $110 \mathrm{lb}$./ sq. in.). Angiogram films were obtained using the Schönander rapid film changer. Pulmonary angiograms were obtained in the control period, after embolization, and after 'therapy'. The E.C.G. (limb lead II), mean pulmonary artery and systemic arterial pressures were continuously monitored and appropriately recorded. Serial studies also included arterial blood gas tensions $\left(\mathrm{PO}_{2}\right.$ and $\left.\mathrm{PCO}_{2}\right)$ and $p \mathrm{Hs}$ using the IL Ultra-Micro $p \mathrm{H}$ and Blood Gas Analysing System, cardiac outputs by a standard dye dilution method, and pulmonary vascular resistances (Motley, 1966).

All animals were then sacrificed and immediately necropsied. The lungs were evaluated pathologically.

\section{RESULTS}

The pulmonary angiogram films shown in Fig. 1 represent typical findings from each of the three groups of animals. The 'after embolism' and '4th hour' films were similar as regards the visible pulmonary arterial filling defects, which sometimes were more apparent on the '4th hour' films. These findings were similar to those of previous studies (Dalen, Mathur, Evans, Haynes, Pur-Shahriari, Stein, and Dexter, 1966). Similarly, in group 2 dogs there were persistent pulmonary arterial filling defects, sometimes more apparent on the "4th hour' films. In contrast, in group 3 dogs the previously appreciable intra-arterial filling defects were significantly decreased on the '4th hour' films. The findings are seen more clearly in Figure 2. Areas of oligaemia are seen on all ' 4 th hour' films, and are more appreciable on the '4th hour' angiogram films of group 3 animals. It is possible that group 3 dogs had more enhanced clot lysis with earlier fragmentation of macroemboli which probably lodged in arteries smaller than second order (diameter 0.1 to $1.0 \mathrm{~mm}$.) as shown by previous studies (Dàlen et al., 1966).

Significant dilatation of the main pulmonary artery and its right and left branches was observed on all 'after embolism' angiograms, and was associated always with significant pulmonary hypertension. The same findings have been reported previously (Lochhead, Roberts, and Dotter, 1952). We attempted to measure this dilatation by selecting on comparable angiograms a $10 \mathrm{sq}$. in. circular area with a fixed mid-reference point (Fig. 3).

${ }^{4}$ Sodium Iothalamate U.S.P. $80 \%$ : Mallinckrodt Pharmaceuticals, St. Louis, Missouri

${ }_{5}$ Model 113, S1, Instrumentation Laboratories, Watertown, Massachusetts 


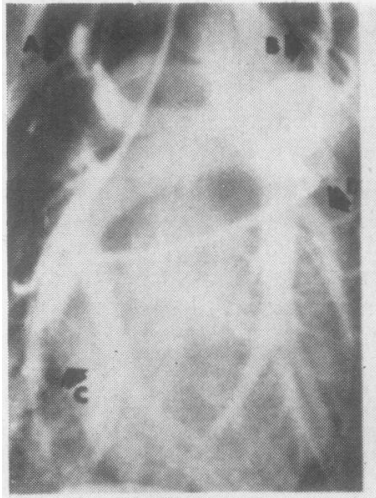

CONTROL m

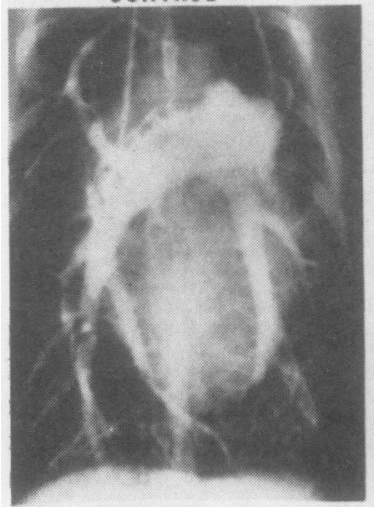

CONTROL O

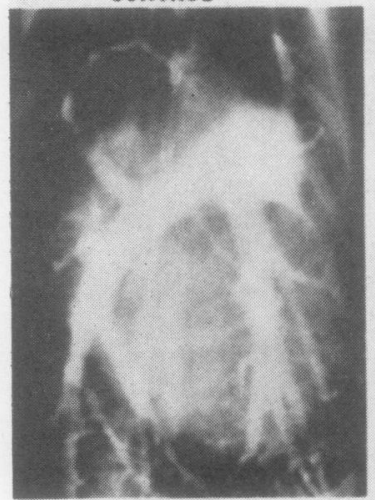

CONTROL I

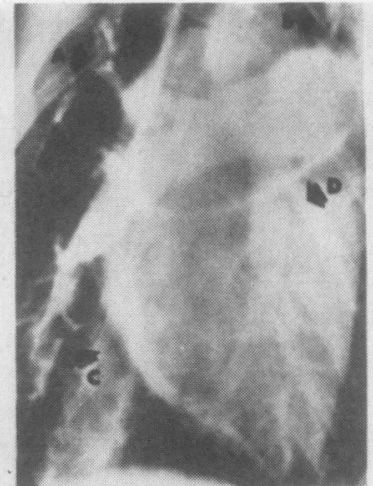

AFTER EMBOLII II

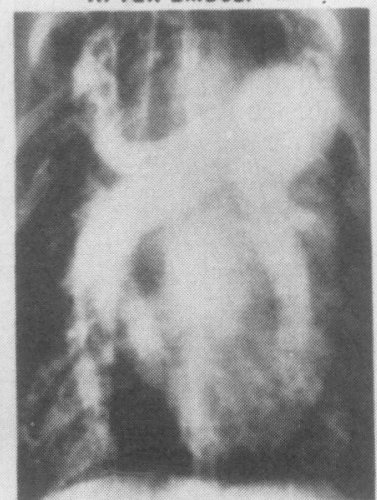

AFTER EMBOLISU O

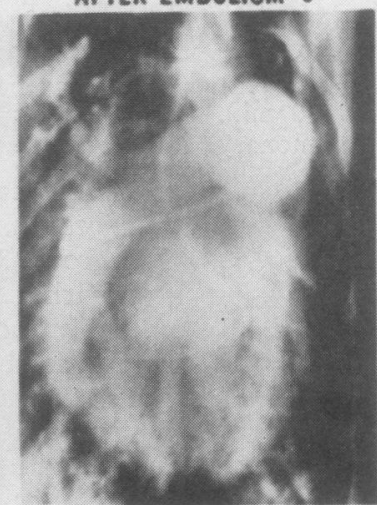

AFTER EMBOLISM $n$

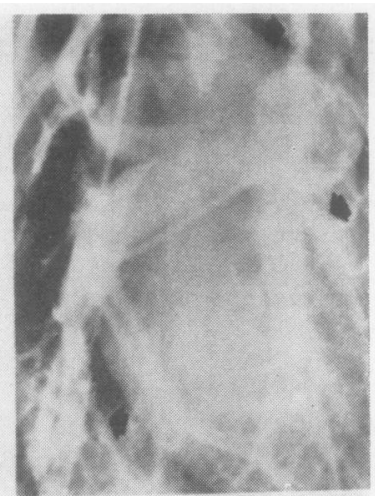

4th HR -05 W

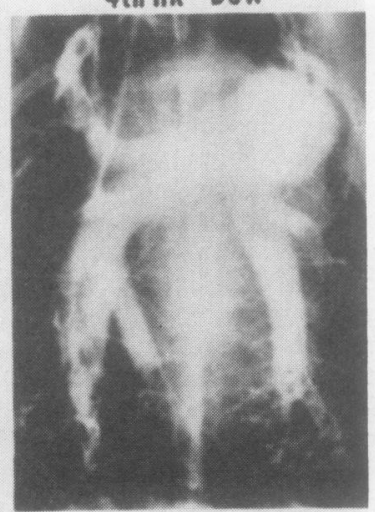

4th HR - IV THR

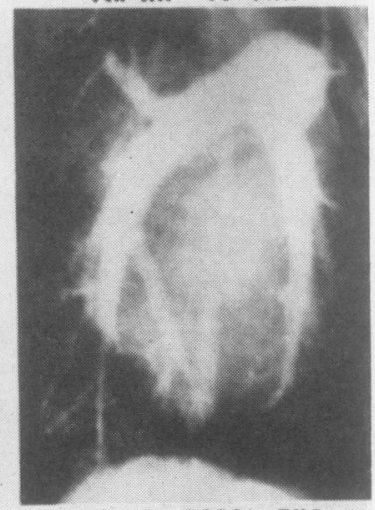

4th HR- LOCAL THR

FIG. 1. Comparable pulmonary angiogram films taken $3 \mathrm{sec}$. after dye injection. Top panel: a typical group 1 animal (D5W), with persistent dilatation of the main pulmonary artery and its right and left branches, and significant pulmonary arterial filling defects, which are more apparent on the '4th hour' films. Middle panel: a typical animal from group 2: again there are persistent vascular dilatations and filling defects. Bottom panel: a typical group 3 animal (Local THR), with filling defects and less vascular dilatation at the fourth hour. 

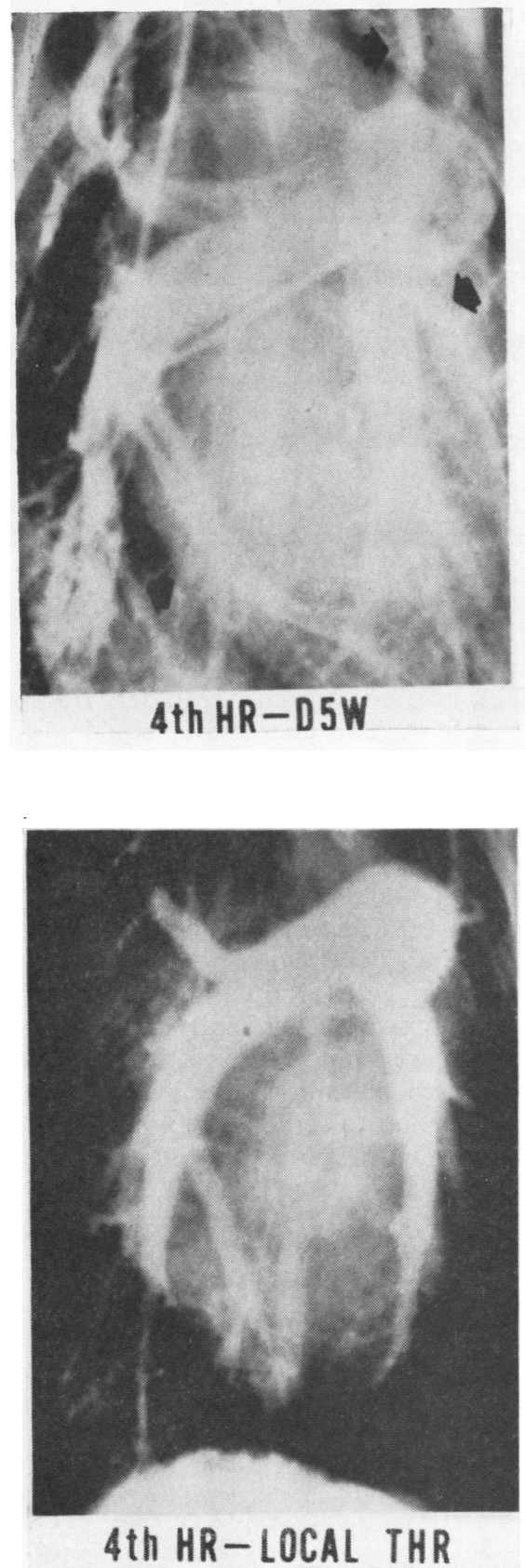

FIG. 2. Enlargements of 4th hour films from Fig. 1. Pulmonary arterial filling defects and vascular dilatation are more apparent in group 1 (DSW) and group 2 (IVTHR) animals. Areas of oligaemia or hyperlucency are more apparent in group 3 (Local THR) dogs (see text for details). There is less evidence of vascular dilatation or intravascular filling defects on this film.

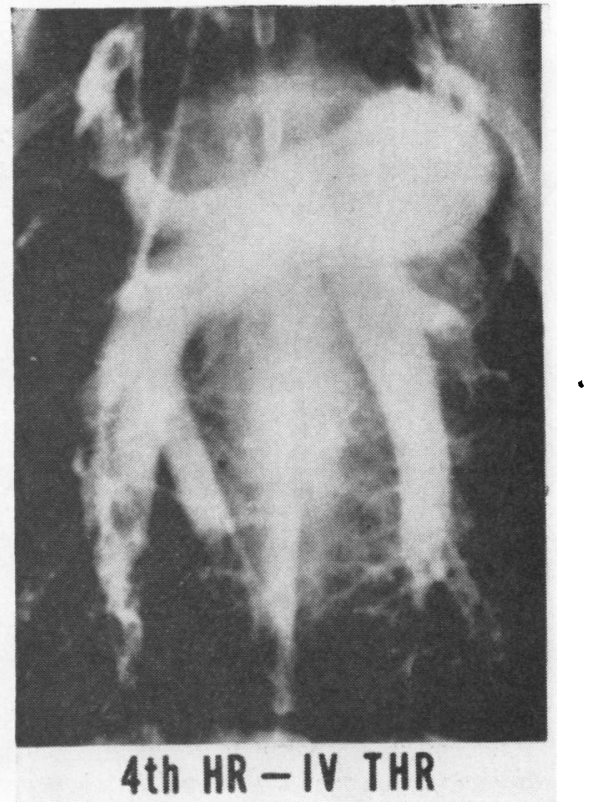

The vascular areas within this circle were then measured with a planimeter, and the measured areas were expressed as per cent changes of control values (Fig. 4).

The '4th hour' angiogram films of group 3 dogs had the least evidence of vascular dilatation. Some dogs in this group had areas actually smaller than their respective control values. This could possibly represent vasoconstriction due to miliary embolism (by microemboli, 8 to $100 \mu$ in diameter) following earlier fragmentation of rapidly lysing macroemboli, 0.17 to $4 \mathrm{~mm}$. in diameter (Dalen, Haynes, Hoppin, Evans, Bhardwaj, and Dexter, 1967). Is the reduction in the vascular areas a reflection of abnormalities secondary to a direct action of Thrombolysin on vascular walls ? Can the temporary presence of a clot cause injury to the vessel walls? These are unanswered questions but still remain as justified speculations. The "4th hour' angiogram films of animals belonging to groups 1 and 2 showed similar findings-comparable and significant vascular dilatation. These two groups were clearly separable from group 3, as shown in Figure 4.

Considering the pulmonary arterial hypertension (Fig. 5), both intravenous and locally instilled Thrombolysin had significantly $(P<0.001)$ beneficial effects (i.e., lowering of the pulmonary hypertension) when compared to locally instilled dextrose $5 \%$ in water solution. In addition, Thrombolysin directly instilled into the main pul- 


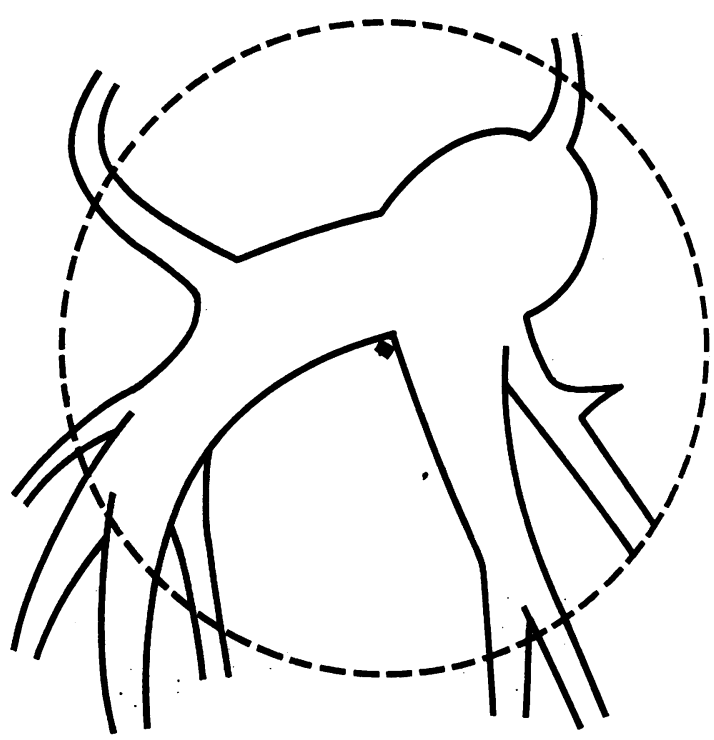

FIG. 3. Vascular areas shown diagrammatically (representing the main pulmonary artery and its right and left branches) within a 10 sq. in. circular area with a fixed mid-reference point were selected and measured by $a$ planimeter. Values were obtained in the control period, after embolization, and after 'therapy'.

monary artery (using half the 'systemic' dose) was significantly more beneficial $(P<0.001)$ than intravenous Thrombolysin (using full 'systemic' doses).

The fall in mean pulmonary artery pressures in groups 1 and 2 did not correlate well with the vascular areas. However, the pressures at the ' 4 th hour' still represent considerable increases com- pared to control values. There is no adequate explanation for this poor correlation. The vascular dilatation is a more reliable indication of significant amounts of intact macroemboli, along with visible intravascular filling defects.

No clear-cut separation of the three groups of animals could be demonstrated with respect to $\overrightarrow{0}$ cardiac outputs, pulmonary vascular resistances, and arterial blood gas tensions $\left(\mathrm{PO}_{2}\right.$ and $\left.\mathbf{P C O}_{2}\right)$ and $p H s$. During and at the end of the fourth hour of study, the results of the arterial blood studies were similar in all animals. The $\mathrm{PO}_{2}$ values ranged from 50 to $60 \mathrm{~mm}$. $\mathrm{Hg}$. The carbon dioxide tensions varied similarly in all dogs $(35$ to $40 \mathrm{~mm}$. $\mathrm{Hg}$ ). Arterial blood $\mathrm{pHs}$ ranged from $7 \cdot 3$ to $7 \cdot 4$. These degrees of hypoxaemia might have altered the pulmonary vascular resistance values. In the dog, hypoxaemia may cause an increase, a decrease, or no effect on calculated pulmonary vascular resistance. Using the dilution technique, the changes in resistance have ranged from -15 to $+20 \%$ (Nahas, Visscher, Mather, Haddy, and Warner, 1954). The anaesthesia could also have played a significant role in producing changes in cardiac output, regional blood distribution, and venous return (Scarborough, 1957).

Heparin was not given just prior to sacrifice of the animals. Dogs belonging to groups 2 and 3 were sacrificed while receiving the last few millilitres of the Thrombolysin solution. Previous studies (Back, Ambrus, Simpson, and Shulman, 1958) have shown that, after lysis of acute clots, reformation was observed in spite of continued treatment with plasmin. These authors attributed this phenomenon to some degree of trauma to the

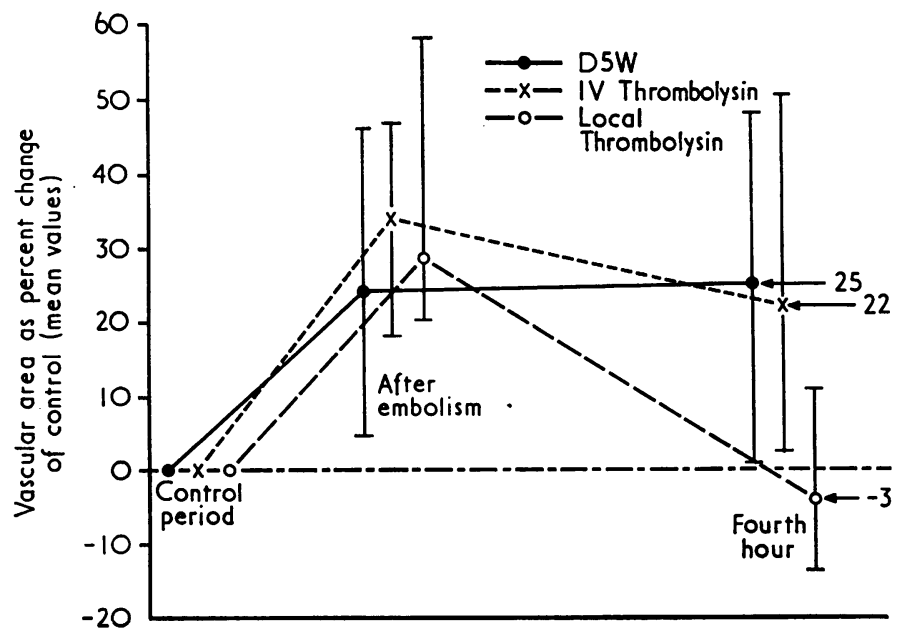

FIG. 4. Graphic representation of the vascular areas expressed as per cent changes of control values. Each point represents the mean with its corresponding range of values. There is a clear-cut separation of group 3 animals from groups 1 and 2 at the fourth hour. 


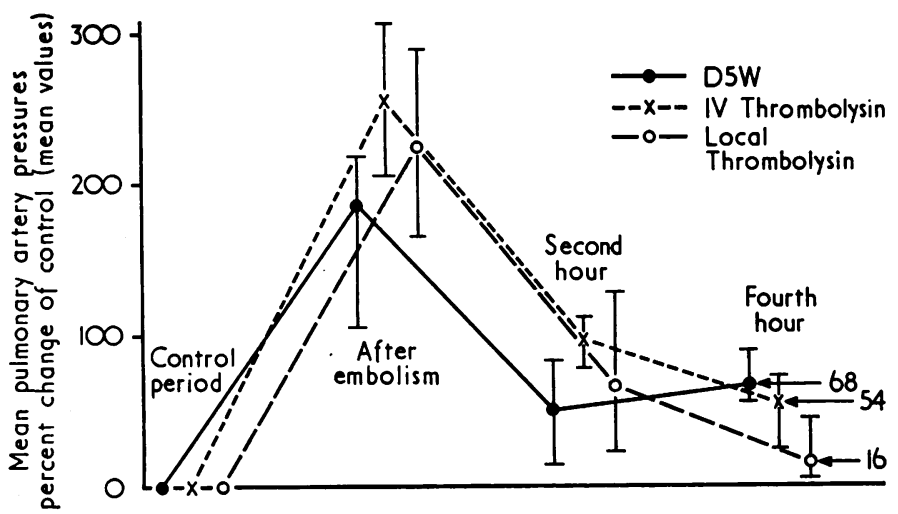

FIG. 5. Graphic representation of the observed mean pulmonary artery pressures expressed as per cent changes of control values. Each point represents the mean with its corresponding range of values. At the fourth hour this graph emphasizes the difference between group 3 animals (Local THR) and groups 1 and 2.

vascular walls. Their experimental design required opening of the wounds daily, but, since trauma was not a factor in our studies, we did not expect this type of clot reformation. However, we expected the formation of so-called propagating red tails (through the red thrombus mechanism) just proximal to macroemboli in all animals.

Pathological studies of the lung were limited to gross and histological examinations of macroemboli. Sections of occluded pulmonary arteries were prepared and stained with haematoxylin and eosin, and in-vitro clots (Figs 7 and 9) were studied in a similar fashion: specific search for microemboli was not made.

Clots seen were located mainly in the lower but occasionally in the upper lobes. The lungs of both group 1 and group 2 animals showed extensive macroembolization. Figure 6 shows an embolus (representative of macroemboli in these two groups of animals) which is intact with no evidence of lysis and resembles an intact in-vitro 'untreated' clot shown in Figure 7. There were no abnormalities of the vascular walls seen by light microscopy.

In contrast. the lungs of animals in group 3 showed remarkably few macroemboli (involvement of only two to three vessels with fragile clots), with easily seen zones of lysis (Fig. 8), most of which were located mainly in the central portions of the clots. These in-vivo findings resembled those seen in Thrombolysin-treated invitro clots (Fig. 9). We are undertaking further histochemical studies (Kwaan and Astrup, 1965).

\section{DISCUSSION}

Reliable evidence is still needed on the effectiveness of fibrinolytic therapy in pulmonary thromboembolism. The basic problem is probably due to the fact that, in the clinical situation, the fate of thrombi and emboli remains obscure.

There is a continuing search for effective thrombolytic agents and for the method of their administration. The studies of Courbier, Torresani, Monties, Patrice, Figarella, and Garabedian (1962) in dogs suggested that fibrinolysis improved the prognosis in pulmonary embolism and, in some animals, brought about complete resolution of pulmonary emboli. Previous studies (Hume, 1961) of several thrombolytic agents have shown unequivocal thrombolysis in several cases. Though effective thrombolytic agents may be at hand, available data concerning the target clot and the inhibitors of the thrombolytic system are still inadequate, and it is not really known why one clot will dissolve and another will not. Most recently, Hirsh, Hale, McDonald, McCarthy, and Cade (1967) reported two patients with acute massive pulmonary embolism who survived after pulmonary arterial infusion of streptokinase. These authors have amply emphasized the ideal treatment of pulmonary embolism: the removal of emboli as quickly and as safely as possible. This needs to be re-emphasized because, of the fatal cases of acute massive pulmonary embolism, the majority (about $50 \%$ ) of patients succumb within the first hour following the embolic episode (Rosenberg, Pearce, and McNulty, 1964), so that many patients die before an operation can be arranged.

Almost all patients with fatal pulmonary embolism have had previous episodes of embolism, and there is serious cardiac dysfunction when emboli occlude more than $60 \%$ of the pulmonary arterial bed. Most of these patients have mixed kinds of emboli-older peripheral clots and relatively recent proximal ones. This latter type of clot probably forms following coating of relatively 


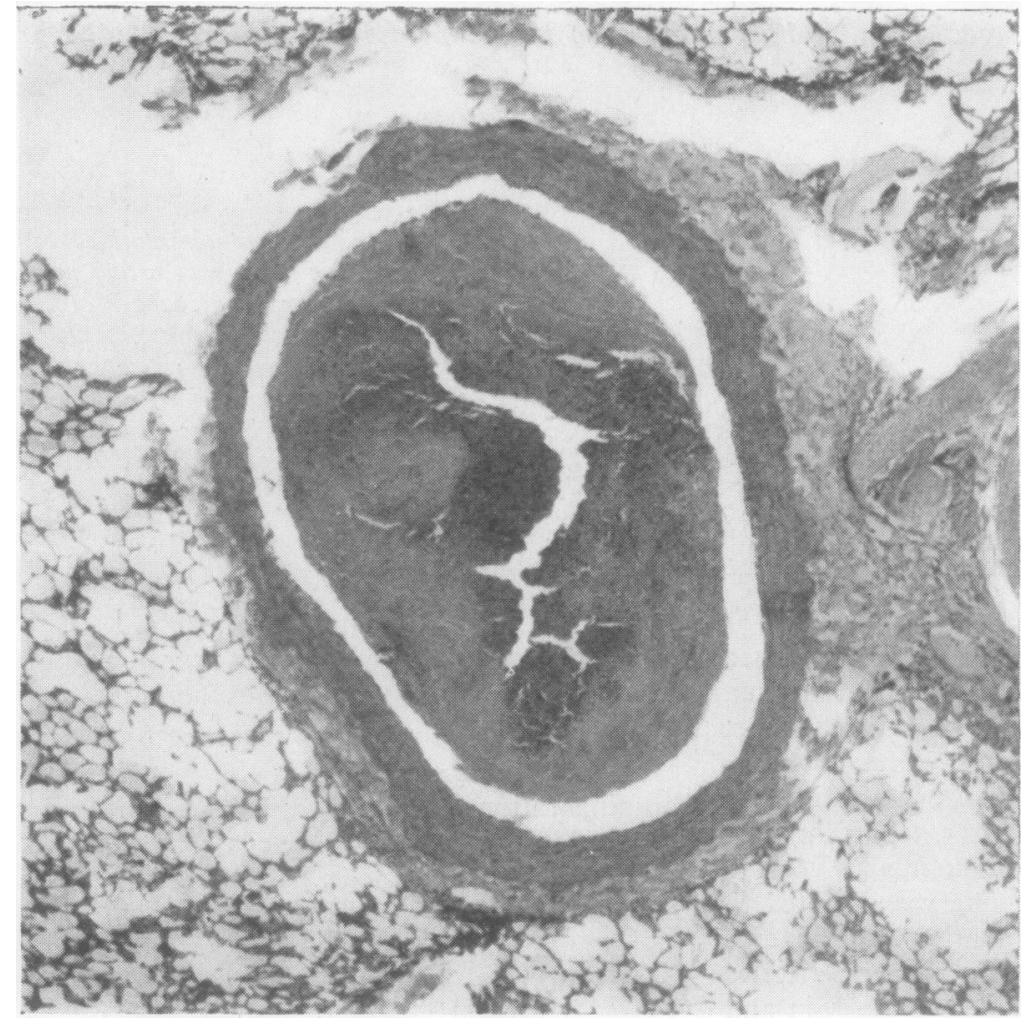

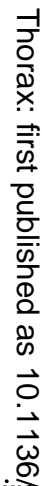

FIG. 6. An in-vivo clot showing its intact quality, and represent ative of macroemboli found in animals belonging to groups it and 2. There is no evidence of lysis. $H$. and $E . \times 40$.

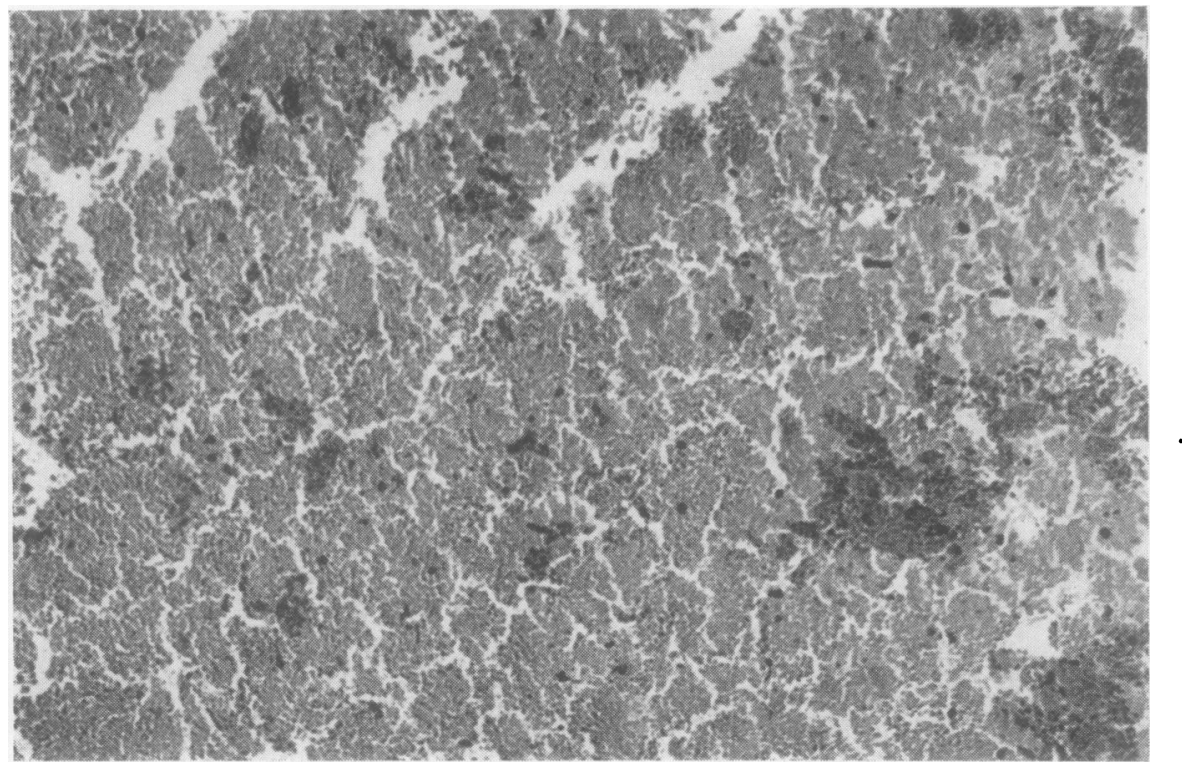

FIG. 7. An 'untreated' in-vitro clot (sampled concurrently from the same batch of preformed autologou令 venous clots used for embolization of a particular greyhound). A sample clot is submerged in dextrose $5 \%$ is water solution and left standing at room temperature for four consecutive hours. By light microscopy it is mon phologically similar to the macroembolus shown in Fig. 6. $H$. and E. $\times 45$. 


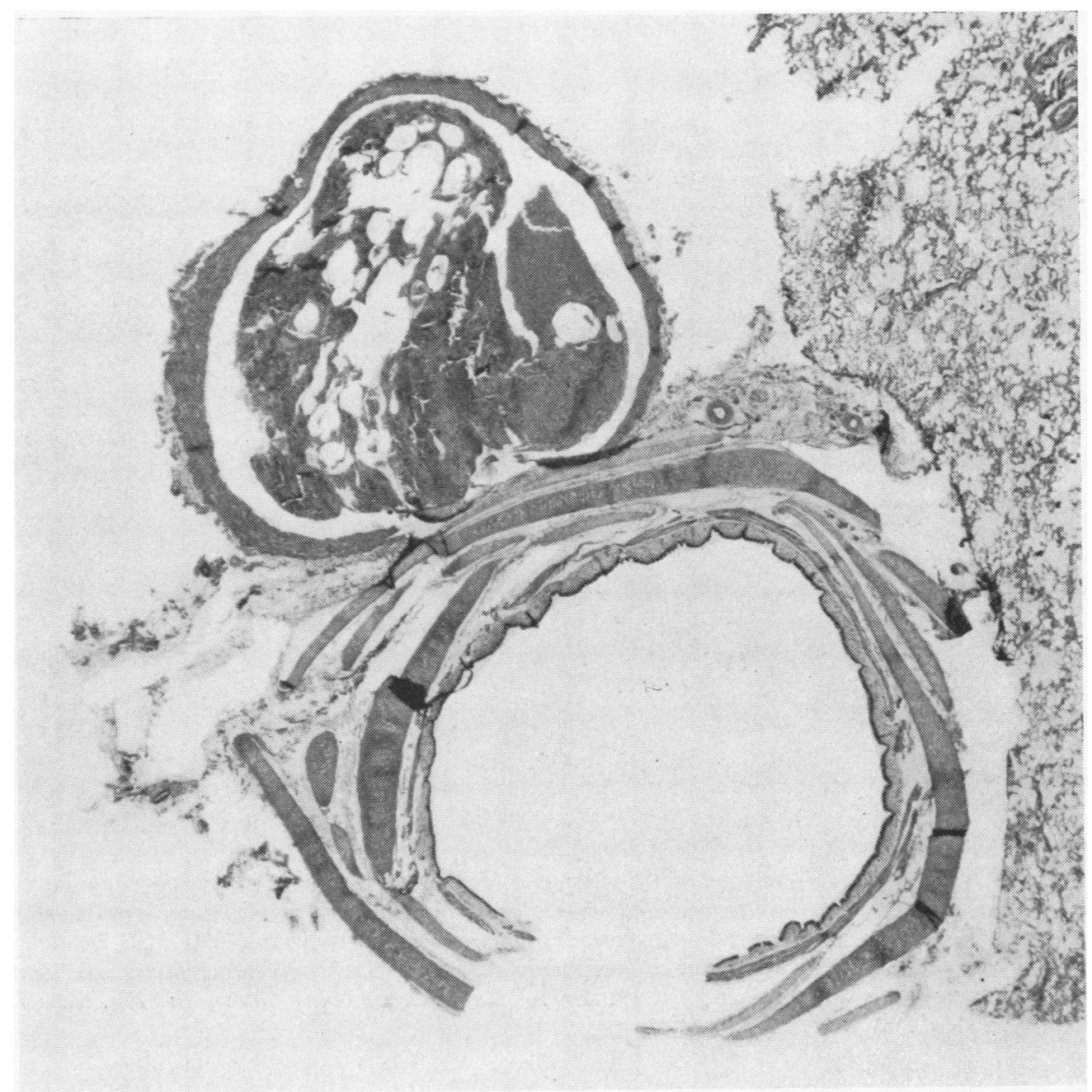

FIG. 8. An in-vivo clot showing its fragmented quality, 'and representative of macroemboli found in dogs belonging to group 3. There are appreciable zones of lysis located mainly in the central portions of the clot, which on gross examination is very friable. $H$. and $E . \times 45$.

older clots by platelets. As the circulation becomes completely occluded, thrombi form through the red thrombus mechanism, resulting in the formation of the so-called propagating red tail (Deykin, 1967). The initial place of thrombolytic therapy in the management of pulmonary embolism then would be to enhance in-vivo lysis of recent clots. Older emboli will probably require more prolonged, carefully monitored administration of thrombolytic agents.

No matter which theory is given preference to explain physiological in-vivo thrombolysis (Alkjaersig, Fletcher, and Sherry, 1959 ; Ambrus and Markus, 1960 ; Fletcher, Alkjaersig, and Sherry, 1959 ; Norman, 1958 ; Sherry, Lindemeyer, Fletcher, and Alkjaersig, 1959), the antiplasminplasmin-clot relationship provides the key to thrombolysis. The plasminogen content of the clot is probably important. The length of time that the clot is in contact with the circulation is equally of major importance.

Several requirements seem of utmost importance and must be satisfied for effective thrombolytic therapy: first, a susceptible clot where fibrin is the principal substrate; second, a susceptible clot containing significant amounts of trapped plasminogen ; and, third, higher concentrations of active enzyme reaching the clot. Presumably, the enzyme will activate plasminogen on the surface of the clot with subsequent diffusion of active moieties into the clot leading to activation of plasminogen around fibrin threads. The clot status determines the lytic ability of enzymes; the older the clot, the less lysis. In 72 hours, the free edges 


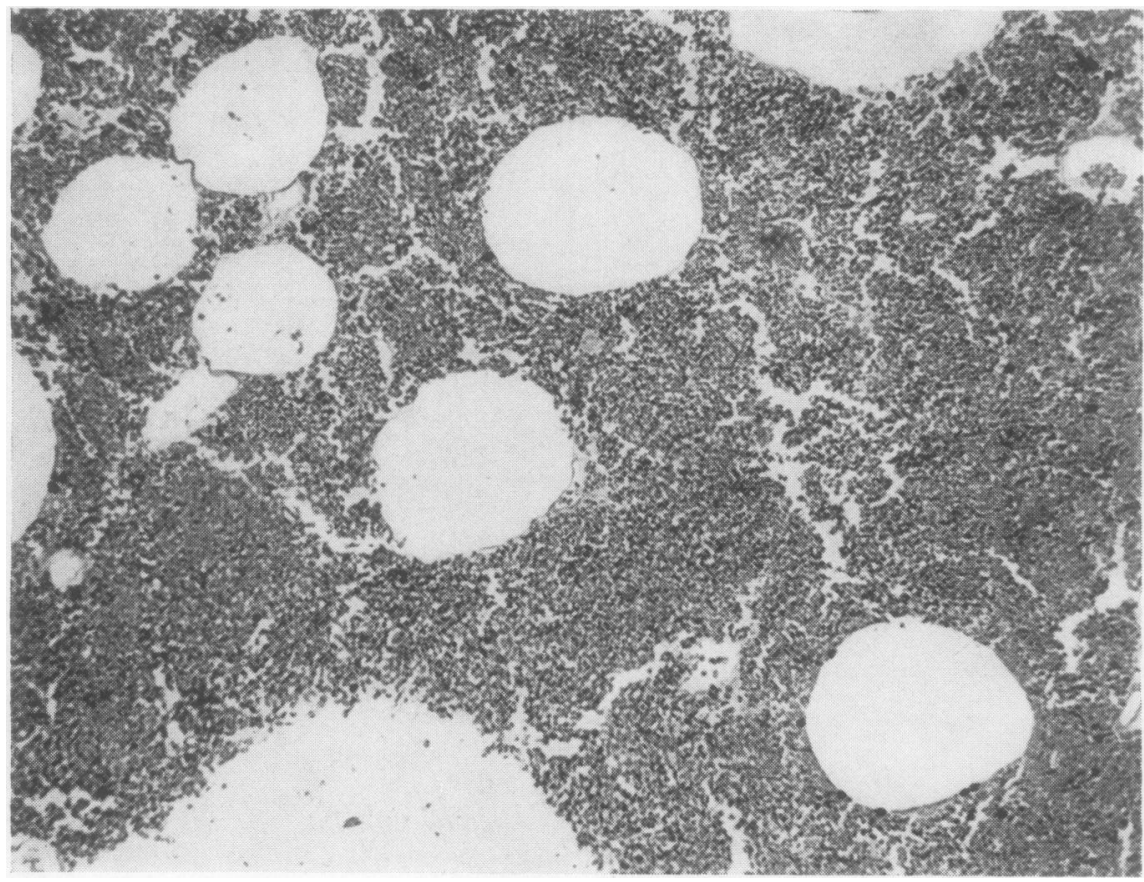

FIG. 9. A 'Thrombolysin-treated' in-vitro clot (sampled concurrently from the same batch of preformed autologous venous clots used for embolization of a particular grehyound). A sample clot is submerged in dextrose $5 \%$ in water solution with 5,000 M.S.D. units of Thrombolysin and left standing at room temperature for four consecutive hours. By light microscopy it is morphologically similar to the macroembolus shown in Fig. 8. H. and $E . \times 45$.

of the clot are covered by endothelial cells (Back, et al., 1958). Williams (1955) noted rapid endothelialization of artificially produced arterial clots occurring in the first 48 hours. This layer of endothelial cells has been shown to be of venous origin and to contain plasminogen activator and has been related to sites of thrombolysis (Kwaan and Astrup, 1965). Nevertheless, this endothelial cell layer may act as a physical barrier between circulating plasmin and the fibrin clot.

Clots used in our study are 24 hours old to satisfy the first requirement for effective thrombolytic therapy. The plasminogen contents of the clots are not known quantitatively.

In the data presented, the direct continuous instillation of Thrombolysin into the main pulmonary artery, in close proximity to the macroemboli, using lower doses than those given into a systemic vein, resulted in more effective lysis of the macroemboli, probably related to the higher concentrations of enzymes reaching the clots. This probably satisfied our third requirement for effective thrombolytic therapy. Though studies of fibrinolysis and coagulation were not done, we think that accelerated lysis of pulmonary macroemboli may be achieved without significant levels of fibrinolytic activity in the systemic vessels.

The continuous instillation of thrombolytic agents into the main pulmonary artery in pulmonary thromboembolism may offer several advantages over systemic intravenous infusion. The data presented in our animals suggest that lower doses can be more effective. The method may prevent significant plasminogen activation in the plasma (where the speed of activation plays a very important role), and may prevent appreciable inhibition of clot lysis (by antiplasmin). Certainly, the importance of inhibitors (playing a central role in any thrombolytic system) and the need to measure them have been emphasized (Johnson and McCarty, 1959) and we re-emphasize this.

If the above assumptions are correct, thrombolytic therapy would be most beneficial during that critical time interval following a massive or sub- 
massive embolic episode when cardiac dysfunction exists, and when enhanced thrombolysis is most necessary to reduce total pulmonary arterial occlusion to less than $60 \%$. Once this is accomplished, prolonged, carefully monitored thrombolytic therapy should lyse all other susceptible clots.

This study was supported in part by grants from the Merck, Sharp, and Dohme Laboratories, West Point, Pennsylvania, and the United Health Fund of Dade County, Florida, U.S.A. The authors are grateful to the paramedical personnel of the Cardiopulmonary Laboratory, Veterans Administration Hospital, Coral Gables, Florida. The authors also acknowledge the generous assistance of Mr. John D. Farrell. The statistical analysis of the data was done by the Biostatistics Division of the University of Miami School of Medicine, Miami, Florida.

\section{REFERENCES}

Alkjaersig, N., Fletcher, A. P., and Sherry, S. (1959). The mechanism of clot dissolution by plasmin. J. clin. Invest., 38, 1086.

Ambrus, C. M., and Markus, G. (1960). Plasmin-antiplasmin complex as a reservoir of fibrinolytic enzyme. Amer. J. Physiol., 199, 491.

Back, N., Ambrus, J. L., Simpson, C. L., and Shulman, S. (1958). Study on the effect of streptokinase-activated plasmin (fibrinolysin) on clots in various stages of organization. J. clin. Invest., 37, 864 .

Courbier, R., Torresani, J., Monties, J. R., Patrice, Y., Figarella, J., and Garabedian, B. (1962). Traitement de l'embolie pulmonaire par la fibrinolysine. Recherches expérimentales. Ann. Chir., 16, 231 .

Dalen, J. E., and Dexter, L. (1967). Diagnosis and management of massive pulmonary embolism. In Disease-a-Month, August, p. 3. Year Book Medical Publishers, Chicago.

- Haynes, F. W., Hoppin, F. G., Jr., Evans, G. L., Bhardwaj, P. and Dexter, L. (1967) Cardiovascular responses to experimental pulmonary embolism. Amer. J. Cardiol., 20, 3.
- Mathur, V. S., Evans, H., Haynes, F. W., Pur-Shahriari, A. A., Stein, P. D., and Dexter, L. (1966). Pulmonary angiography in experimental pulmonary embolism. Amer. Heart J., 72, 509.

Deykin, D. (1967). Thrombogenesis. New Engl. J. Med., 276, 622.

Fletcher A. P., Alkjaersig, N., and Sherry, S. (1959). The maintainance of a sustained thrombolytic state in man. I. Induction and effects. J. clin. Invest., 38, 1096.

Hirsh, J. Hale, G. S., McDonald, I. G., McCarthy, R. A., and Cade J. F. (1967). Resolution of acute massive pulmonary embolism after pulmonary arterial infusion of streptokinase. Lancet, $2,593$.

Hume, M. (1961). Thrombolysis of the experimental radioactive pulmonary embolus. New Engl. J. Med., 264, 471 .

Johnson, A. J., and McCarty, W. R. (1959). The lysis of artificially induced intravasculsr clots in man by intravenous infusions of streptokinase. J. clin. Invest., 38, 1627.

Kwaan, H. C., and Astrup, T. (1965). Fibrinolytic activity in thrombosed veins. Circulat. Res., 17, 477.

Lochhead, R. P., Roberts, D. J., and Dotter, C. T. (1952). Pulmonary embolism. Experimental angiocardiographic study. Amer. $J$. Roentgenol., 68, 627.

Magtira, D., Winkler, J., Duprey, R., and Baum, G. L. (1966). Bedside cardiopulmonary evaluation. Clin. Res., 14, 42.

Motley, H. L. (1966). The pulmonary circulation. In Intravascular Catheterization. Ed. Zimmerman, H. A., 2nd ed., p. 910. Thomas, Springfield, Illinois.

Nahas, G. G., Visscher, M. B., Mather, G. W., Haddy, F. J., and Warner, H. R. (1954). Influence of hypoxia on the pulmonary circulation of nonnarcotized dogs. J. appl. Physiol., 6, 467.

Norman, P. S. (1958). Studies of the plasmin system. II. Inhibition of plasmin by serum or plasma. J. exp. Med., 108, 53.

Rosenberg, D. M. L., Pearce, C., and McNulty, J. (1964). Surgical treatment of pulmonary embolism. J. thorac. cardiovasc. Surg.,

Scarborough, W. R. (1957). Some circulatory effects of morphinebarbiturate anaesthesia, artificial respiration, and abdominal compression based on ballistocardiographic observations on dogs. Amer. Heart J., 54, 651.

Sherry, S,. Lindemeyer, R. I., Fletcher, A. P., and Alkjaersig, N. (1959). Studies on enhanced fibrinolytic activity in man. J. clin. Invest. $38,810$.

Wessler, S., Freiman, D. G., Ballon, J. D., Katz, J. H., Wolff, R., and Wolf, E. (1961). Experimental pulmonary embolism with serum-induced thrombi. Amer. J. Path., 38, 89.

Williams, G. (1955). Experimental arterial thrombosis. J. Path. Bact., $69,199$. 